\title{
Regularization method for complete singular integral equation with Hilbert kernel on open arcs
}

\author{
Li-xia Cao ${ }^{1, \text { a }}$ \\ ${ }^{1}$ Mathematics College, Northeast Petroleum University, Daqing, Heilongjiang 163318, China \\ acaolixia98237@163.com
}

Keywords: regularization method; Hilbert kernel; singular integral equation; open arcs

Abstract. We considered the regularization method for a kind of complete singular integral equation with Hilbert kernel on open arcs lying in a period strip. And based on this, we obtained the solvable Noether theorem for this kind of complete singular integral equations.

\section{Introduction}

In $[1,2,3,4,5]$, the authors discussed the singular integral equation with Cauchy kernel on the real half-line on the real line, or on a complicated contour. In [6], the authors discussed Numerical solution of a singular integral equation with Cauchy kernel in the plane contact problem. While the solvable Noether theorem for complete singular integral equation with Hilbert kernel on open arcs, has few been discussed. Here we discuss a kind of complete singular integral equation with Hilbert kernel on open arcs by considering the regularization method, and give the solvable Noether theorem.

\section{The complete singular integral equation with Hilbert kernel on open arcs}

Suppose that $L=\sum_{j} L_{j}$ is a finite set of nonintersecting open arcs, with period $a \pi$ lying entirely in the in the same periodic region $S:|\operatorname{Re} z|<a \pi / 2$, and being positively oriented. We consider the following the singular integral equation with Hilbert kernel for a Hölder continuous function $\varphi(t)$ on $L$

$$
K \varphi \equiv A\left(t_{0}\right) \varphi\left(t_{0}\right)+\frac{1}{a \pi i} \int_{L} K\left(t_{0}, t\right) \cot \frac{t-t_{0}}{a} \varphi(t) d t=f\left(t_{0}\right), t_{0} \in L .
$$

where $A(t), B(t), f(t) \in H(L), K\left(t_{0}, t\right) \in H_{0}(L \times L)$ are given functions with $A^{2}(t)-B^{2}(t) \neq 0$.We assume the different points $C_{1}, C_{2}, \cdots, c_{n}$ are the all nodes on $L$ (including the end-points of $L_{j}$ and the discontinuity points of $A(t), B(t)$ and $f(t))$. Without loss of generality, no special condition is needed except for the requirement that $\varphi \in h=h\left(c_{1}, c_{2}, \cdots, c_{q}\right)(q \leq n)$, that is $\varphi$ is bounded near $c_{1}, c_{2}, \cdots$ and $c_{q}$, while $\varphi$ has a singularity of order less than 1 at any other nodes. For $L$, we permit it to arrive at the boundary of the region $S$, and in this case we regarded $c$ and $c \pm a \pi$ as the same one.

If we let $B(t)=K(t, t)$, then (1) can be rewritten as

$$
K \varphi \equiv A\left(t_{0}\right) \varphi\left(t_{0}\right)+\frac{B\left(t_{0}\right)}{a \pi i} \int_{L} \cot \frac{t-t_{0}}{a} \varphi(t) d t+\int_{L} k\left(t_{0}, t\right) \varphi(t) d t=f\left(t_{0}\right), t_{0} \in L,
$$

where $k\left(t_{0}, t\right)=\left[K\left(t_{0}, t\right)-K(t, t)\right] \cot \left[\left(t-t_{0}\right) / a\right]$. Since $K\left(t_{0}, t\right) \in H^{\alpha}(0<\alpha \leq 1)$, we have $\left|k\left(t_{0}, t\right)\right| \leq A /\left|t-t_{0}\right|^{\lambda}(0 \leq \lambda=1-\alpha<1)$, and now $k \varphi \equiv \int_{L} k\left(t_{0}, t\right) \varphi(t) d t$ is an F-I operator, and

$$
K^{0} \varphi \equiv A\left(t_{0}\right) \varphi\left(t_{0}\right)+\frac{B\left(t_{0}\right)}{a \pi i} \int_{L} \cot \frac{t-t_{0}}{a} \varphi(t) d t,
$$

is actually the characteristic operator of operator $K$. Therefore, Eq.(1) can be rewritten as

$$
\left(K^{0}+k\right) \varphi=f .
$$

The associative equation of Eq. (1) takes the form 


$$
K^{\prime} \psi \equiv A\left(t_{0}\right) \psi\left(t_{0}\right)-\frac{1}{a \pi i} \int_{L} B(t) \psi(t) \cot \frac{t-t_{0}}{a} d t \pm \int_{L} k\left(t, t_{0}\right) \psi(t) d t=g\left(t_{0}\right)
$$

Let $\kappa_{j}=[\arg \{[A(t)-B(t)] /[A(t)+B(t)]\}]_{L_{j}} /(2 \pi)$, then the index of Eq. (1) is $\kappa=\sum_{j=1}^{p} \kappa_{j}$.

\section{Regularization method}

We may rewrite (2) as follows

$$
K^{0} \varphi=f\left(t_{0}\right)-k \varphi .
$$

when $t_{0}$ and $t_{1}$ are on the same smooth arc (including endpoints), we have

$$
\left|k\left(t_{0}, t\right)\right|=\left|\left[K\left(t_{0}, t\right)-K\left(t_{0}, t_{0}\right)\right] \cot \frac{t-t_{0}}{a}\right| \leq A\left|t-t_{0}\right|^{\alpha}\left|\frac{a}{t-t_{0}}+\sum_{n=1}^{\infty} b_{n}\left(t-t_{0}\right)^{n}\right| \leq \frac{M}{\left|t-t_{0}\right|^{\lambda}}(0 \leq \lambda=1-\alpha<1),
$$

so $\int_{L} k\left(t_{0}, t\right) \varphi(t) d t$ is an F-I operator, and $k\left(t_{0}, t\right) \in H_{0}(L)$, therefore $f-k \varphi \in H_{0}$. Let

$$
X(z)=\prod_{j=1}^{p}\left(\tan \frac{Z}{a}-\tan \frac{C_{j}}{a}\right)^{\lambda_{j}} e^{\Gamma(z)} \text { and } K^{*} f \equiv A^{*}\left(t_{0}\right) f\left(t_{0}\right)-\frac{B^{*}\left(t_{0}\right) Z\left(t_{0}\right)}{a \pi i} \int_{L} \frac{f(t)}{Z(t)} \cot \frac{t-t_{0}}{a} d t .
$$

If $\kappa \geq 0$, then $\varphi$ must satisfies

$$
\varphi\left(t_{0}\right)=K^{*}(f-k \varphi)+B^{*}\left(t_{0}\right) Z\left(t_{0}\right) P_{k}\left(\tan \frac{t_{0}}{a}\right),
$$

that is

$$
\varphi\left(t_{0}\right)+K^{*} k \varphi=f^{*}\left(t_{0}\right)
$$

where

$$
f^{*}\left(t_{0}\right)=K^{*} f+B^{*}\left(t_{0}\right) Z\left(t_{0}\right) P_{\kappa}\left(\tan \frac{t_{0}}{a}\right) .
$$

Obviously, $f^{*}\left(t_{0}\right) \in h$. Conversely, if $\varphi \in h$ (for some $P_{\kappa}$ ) satisfies (7)', then $\varphi$ is the solution for (2).

If $\kappa<0$, if and only if

$$
\left\{\begin{array}{l}
\int_{L}[f(t)-k \varphi]\left(\tan ^{j-1} \frac{t}{a}+\tan ^{j+1} \frac{t}{a}\right) / Z(t) \mathrm{d} t=0, \quad j=1, \cdots,-\kappa-1, \\
\int_{L}[f(t)-k \varphi]\left(\sin _{\frac{v}{2}}-\cos \frac{v}{2} \tan \frac{t}{a}\right) / Z(t) \mathrm{d} t=0
\end{array}\right.
$$

Eq.(7) has solution in class $h$, and its unique solution is $\varphi\left(t_{0}\right)=K^{*}(f-k \varphi)$, that is (7)' with $P_{\kappa} \equiv 0$.

Since $k$ is a Fredholm operator, we have

$$
\int_{L} k \varphi\left[\tan ^{j-1} \frac{t}{a}+\tan ^{j+1} \frac{t}{a}\right] / Z(t) d t=\int_{L} \varphi(t) k^{\prime}\left[\tan ^{j-1} \frac{t}{a}+\tan ^{j+1} \frac{t}{a}\right] / Z(t) d t, j=1, \cdots,-\kappa-1
$$

thus

$$
\left\{\begin{array}{l}
\int_{L}\left[\tan ^{j-1} \frac{t}{a}+\tan ^{j+1} \frac{t}{a}\right] f(t) / Z(t) \mathrm{d} t=\int_{L} \varphi(t) P_{j}(t) \mathrm{d} t, \quad j=1, \cdots,-\kappa-1, \\
\int\left[\sin _{L} \frac{v}{2}-\cos \frac{v}{2} \tan \frac{t}{a}\right] f(t) / Z(t) \mathrm{d} t=\int_{L} \varphi(t) P_{0}(t) \mathrm{d} t
\end{array}\right.
$$

where

$$
\begin{gathered}
P_{j}(t)=k^{\prime}\left\{\left[\tan ^{j-1} \frac{t}{a}+\tan ^{j+1} \frac{t}{a}\right] / Z(t)\right\}=\int_{L}\left[\tan ^{j-1} \frac{t_{1}}{a}+\tan ^{j+1} \frac{t_{1}}{a}\right] / Z(t) d t_{1}, j=1, \cdots,-\kappa-1, \\
P_{0}(t)=k^{\prime}\left\{\left[\sin \frac{v}{2}-\cos \frac{v}{2} \tan \frac{t}{a}\right] / Z(t)\right\}=\int_{L} k\left(t_{1}, t\right)\left[\sin \frac{v}{2}-\cos \frac{v}{2} \tan \frac{t_{1}}{a}\right] / Z(t) \mathrm{d} t_{1},
\end{gathered}
$$

and $P_{j}(t)(j=0,1, \cdots, \kappa-1)$ are the known functions in class $H_{0}$, and when $\kappa \leq 0$ the Eq. (2) in class $h$ is equivalent to Eq. (7) ' in class $h$ (with $P_{\kappa} \equiv 0$ in (8)) and the additive condition (9) or (9)' by the same reason to the case that when $\kappa \geq 0$. 
Let us demonstrate $K^{*} k$ is a weak Fredholm operator. Since $K^{*} f \equiv A^{*}\left(t_{0}\right) f\left(t_{0}\right)-\frac{B^{*}\left(t_{0}\right) Z\left(t_{0}\right)}{a \pi i} \int_{L} \frac{f(t)}{Z(t)} \cot \frac{t-t_{0}}{a} \mathrm{~d} t$,

we have $K^{*} k \varphi=A^{*}\left(t_{0}\right)(k \varphi)\left(t_{0}\right)-\frac{B^{*}\left(t_{0}\right) Z\left(t_{0}\right)}{a \pi i} \int_{L} \cot \frac{t-t_{0}}{a} \frac{\mathrm{d} t}{Z(t)} \cdot \int_{L} k\left(t, t_{1}\right) \varphi\left(t_{1}\right) d t_{1}$.

Since the order of Hilbert integral and common integral can be exchanged, we obtain

$$
K^{*} k \varphi=A^{*}\left(t_{0}\right)(k \varphi)\left(t_{0}\right)-B^{*}\left(t_{0}\right) Z\left(t_{0}\right) \int_{L} k^{*}\left(t_{0}, t_{1}\right) \varphi\left(t_{1}\right) d t_{1},
$$

where $k^{*}\left(t_{0}, t\right)=\frac{1}{a \pi i} \int_{L} k\left(t, t_{1}\right) \cot \left(\frac{t-t_{0}}{a}\right) / Z(t) d t$ has at the most less than one order singularity at the node $t_{0}$ and so that it is a weak Fredholm kernel.

The following is the discussion about regularization method for Eq.(2).

We write (7)' as follows

$$
\varphi+K^{*} k \varphi \equiv \varphi\left(t_{0}\right)+\int_{L} N\left(t_{0}, t\right) \varphi(t) d t=f^{*}\left(t_{0}\right)
$$

where (following from (10) )

$$
N\left(t_{0}, t\right)=A^{*}\left(t_{0}\right) k\left(t_{0}, t\right)-\frac{B^{*}\left(t_{0}\right) Z\left(t_{0}\right)}{a \pi i} \int_{L} \frac{k\left(t_{1}, t\right)}{Z\left(t_{1}\right)} \cot \frac{t_{1}-t_{0}}{a} d t_{1} .
$$

Now the associative equation of (11) is

$$
\psi+k^{\prime} K^{*^{\prime}} \psi \equiv \psi\left(t_{0}\right)+\int_{L} N\left(t, t_{0}\right) \psi(t) d t=0
$$

and from (12), we know $N\left(t, t_{0}\right)=A^{*}\left(t_{0}\right) k\left(t, t_{0}\right)-\frac{B^{*}\left(t_{0}\right) Z\left(t_{0}\right)}{a \pi i} \int_{L} \frac{k\left(t_{1}, t_{0}\right)}{Z\left(t_{1}\right)} \cot \frac{\left(t_{1}-t\right)}{a} d t_{1}$.

In order to apply Fredholm Theorem, we try to convent (2.36) into the case with finite kernel. From properties of $(7)$ and $X^{+}(t)$ we have $Z(t)=\prod_{j=1}^{n}\left(\tan \frac{t}{a}-\tan \frac{c_{j}}{a}\right)^{\lambda_{j}} m(t)$, where $m(t) \in H_{0}(L)$ and $m(t) \neq 0$. When $c_{j} \in\left\{c_{1}, \cdots, c_{q}\right\}$, we have $0<\operatorname{Re} \gamma_{j}<1$, when $c_{j}$ is a special node, $\operatorname{Re} \gamma_{j}=0$, and when $c_{j}$ is one of the other common nodes such as $c_{q+1}, \cdots, c_{m}$, we have $-1<\operatorname{Re} \gamma_{j}<0$. Here we denote these special nodes of $\operatorname{Re} \gamma_{j}=0 \quad$ as $c_{p+1}, \cdots, c_{n}(p \geq m)$. Let $T(t)=\prod_{j=q+1}^{m}\left(\tan \frac{t}{a}-\tan \frac{c_{j}}{a}\right)^{\gamma_{j}} \prod_{j=p+1}^{n}\left[\log \left(t-c_{j}\right)+d_{j}\right] \quad, \quad$ then for any $j \in\{p+1, \cdots, n\} \quad$ we have $\log \left(t-c_{j}\right)+d_{j} \neq 0$ on $L$, which guarantee that $1 / T(t)$ is bounded on $L$.

Now we introduce a new unknown function $\varphi_{0}(t)=\varphi(t) / T(t)$, then Eq.(7)' becomes

$$
\varphi_{0}\left(t_{0}\right)+\int_{L} N_{0}\left(t_{0}, t\right) \varphi_{0}(t) T(t) d t=f_{0}^{*}\left(t_{0}\right),
$$

where $f_{0}^{*}(t)=f^{*}(t) / T(t)$ and $N_{0}\left(t_{0}, t\right)=N\left(t_{0}, t\right) / T\left(t_{0}\right)$, where $N_{0}\left(t_{0}, t\right)$ is bounded. By substitution $\tau=\int_{c}^{t} T(t) d t\left(t \in L_{j}\right)$, we have $d \tau=T(t) d t$ and Eq.(14) becomes

$$
\varphi_{0}\left(\tau_{0}\right)+\int_{\wedge} N_{0}\left(\tau_{0}, \tau\right) \varphi_{0}(\tau) d \tau=f_{0}^{*}\left(\tau_{0}\right), \tau_{0} \in \wedge,
$$

where $\wedge$ is the image of $L$ under the transformation, and Eq.(15) is actually a Fredholm equation with bounded kernel, $f_{0}^{*}\left(\tau_{0}\right)$ is also bounded.

Following the former transformation, the homogeneous associative equation (13) becomes

$$
\psi\left(\tau_{0}\right)+\int_{\wedge} N_{0}\left(\tau_{0}, \tau\right) \psi(\tau) d \tau=0
$$

Applying Fredholm Theorem to Eq. (15) and (16) denoting by the original variables $t$ and $t_{0}$,we obtain the following results.

Theorem 3.1 Equation (11) is solvable in class $h$ if and only if the condition

$$
\int_{L} f^{*}(t) w_{j}(t) d t=0, j=1,2, \cdots, v
$$


is satisfied, where $\left\{w_{j}(t)\right\}_{1}^{v}$ is the set of linearly independent solutions of the homogeneous associative equation $w\left(t_{0}\right)+k^{\prime} K^{* \prime} w=0$ in class $h^{\prime}$.

When the solvable condition (17) is satisfied, the solution of (11) in class $h$ can be written as

$$
\varphi\left(t_{0}\right)=\Gamma f^{*}+\sum_{j=1}^{v} C_{j} x_{j}\left(t_{0}\right),
$$

where $C_{1}, \cdots, C_{v}$ are arbitrary complex constants, $\left\{\chi_{j}(t)\right\}_{1}^{v}$ is the set of linearly independent solutions of the homogeneous equation of Eq.(11), while $\Gamma f^{*} \equiv f^{*}\left(t_{0}\right)+\int_{L} \Gamma\left(t_{0}, t\right) f^{*}(t) d t$, and $\Gamma\left(t_{0}, t\right)$ is the generalized resolvent kernel.

\section{Noether theorem of a kind of singular integral equation with Hilbert kernel on open arcs}

Applying the above results and following [1], we may obtain the following result.

\section{Theorem 4.1 (Noether Theorem)}

(a) The numbers of the linearly independent solutions for the homogeneous Eq. (2) ( $f \equiv 0$ )in class $h$ and for the homogeneous associative Eq. $K^{0^{\prime}} \psi=0$ in $h^{\prime}$ are all finite, denoted by $l$ and $l^{\prime}$ respectively. Moreover $l-l^{\prime}=\kappa$, where is the index of Eq.(2) in class $h$.

(b) Eq.(2) is solvable in class $h=h\left(c_{1}, \cdots, c_{q}\right)$ if and only if the condition

$$
\int_{L} f \psi_{j} d t=0, j=1, \cdots, l^{\prime}
$$

is satisfied, where $\left\{\psi_{j}\right\}^{l^{\prime}}$ is the set of linearly independent solutions in $h^{\prime}$ of equation $K^{0^{\prime}} \psi=0$.

\section{References}

[1] Lu J K.,Boundary Value Problems for Analytic Functions. World Sci Publ, Singapore(2004)

[2] D. Pylak; R. Smarzewski; M. A. Sheshko, A Singular Integral Equation with a Cauchy Kernel on the Real Half-Line, J. Differential Equations, Volume 41(2005) 1775-1788.

[3]B. G. Gabdulkhaevt; I. N. Tikhonov, Methods for Solving a Singular Integral Equation with Cauchy Kernel on the Real Line,J. Differential equations, Volume 44(2008) 980-990.

[4] M. A. Sheshko; S. M. Sheshko, Singular integral equation with Cauchy kernel on the real axis, J. Differential Equations, Volume 46(2010) 568-585.

[5] M. A. Sheshko; S. M. Sheshko, Singular integral equation with Cauchy kernel on a complicated contour, Differential Equations, Volume 47(2011) 1344-1356.

[6] M. R. Capobianco; G. Criscuolo, Numerical solution of a singular integral equation with Cauchy kernel in the plane contact problem,J. Quarterly of Applied Mathematics, Volume 69(2011) 79-89. 